\title{
Spectrum Allocation for Hyper-Dense Small Cell Networks: A Partially-Distributed Approach
}

\author{
Junfei Qiu ${ }^{1, a^{*}}$, Zhiyong Du, ${ }^{2, b}$, Youming Sun ${ }^{3, c}$ and Ducheng $\mathrm{Wu}^{4, \mathrm{~d}}$ \\ ${ }^{1,2,4}$ College of Communications Engineering, PLA University of Science and Technology, Nanjing, \\ China, 210007 \\ ${ }^{3}$ National Digital Switching System Engineering and Technological Research Center, Zhengzhou, \\ China, 450000 \\ a ${ }^{*}$ junfeiqiu@163.com, bduzhiyong2010@gmail.com, 'sunyouming10@163.com, \\ dwuducheng@foxmail.com
}

\begin{abstract}
Keywords: Small cells; Spectrum allocation; Clustering; Q-learning
Abstract. In this paper, we investigate the problem of spectrum allocation for small cell networks with dense deployment of access points in large quantities. Since the formulated problem is intractable in the context of large-scale networks, finding the optimal solution is very complex. To tackle this issue, here, we develop a partially-distributed approach to achieve desirable performance. Specifically, the proposed scheme mainly consists of three phases: dividing network into disjoint clusters, sub-channel assignment within each cluster and interference elimination among clusters. Finally, numerical results show that the partially-distributed solution outperforms some existing approaches. Meanwhile, the proposed scheme can achieve desirable performance, which is close to that of the global optimization method, with a faster convergence speed.
\end{abstract}

\section{Introduction}

Small cells have been regarded as an emerging approach to providing high throughput for existing cellular networks [1]. Furthermore, it is expected that small cells such as femtocells are to be densely deployed in large quantities in the near future [2]. However, with the large-scale deployment of such low-power and low-cost small cell access points (SAPs), cells interference becomes more and more serious. Therefore, effective spectrum allocation is crucial.

Several spectrum allocation solutions for small cell networks in recent studies have been proposed, using e.g., centralized assignment approach [3], graph-based model [4], cognitive radio aided scheme [5] and cooperative strategy based on coalitional game [6]. Nevertheless, to the best of our knowledge, few approaches can scale to dense scenario, which necessitates new analysis methods for effective spectrum allocation in large-scale small cell networks.

In this paper, we focus on spectrum allocation techniques in dense small cell networks and propose a partially-distributed scheme, which can be suitable for large-scale network scenarios. To begin with, in terms of a large-scale network, we resort to the clustering method which can divide the network into smaller modules. Then, for a given cluster configuration, within each cluster, one SAP is elected as a cluster head $(\mathrm{CH})$ to assign sub-channels among small cells in that cluster and a low-complexity graph coloring based spectrum allocation algorithm is presented. Next, to further mitigate the mutual interference among neighboring clusters, a decentralized local Q-learning algorithm is proposed. Finally, the effectiveness of the proposed solution is verified by simulation results.

\section{System Model and Problem Formulation}

We consider a downlink transmission with dense deployment of $K$ SAPs in the context of OFDMA-based small cell network. We denote by $\mathbf{F}=\left\{F_{1}, F_{2} \mathrm{~K} F_{K}\right\}$ the set of SAPs and $|\mathbf{F}|=K$. Let $\mathbf{N}=\{1,2 \mathrm{~K} N\}$ denote the set of sub-channels in the system and $|\mathbf{N}|=N$. Here, we adopt an orthogonal channel assignment between small cells and the macrocell that eliminates the cross-layer interference. 
As in [7] and [8], we assume that at most one user equipment (UE) is active within the coverage of the SAP at the time-instant considered.

We denote by $p_{T}$ the transmission power of SAPs. Let $\boldsymbol{\eta}=\left[\eta_{i}^{n}\right]$ with size of $N \times K$ be the sub-channel allocation matrix and $\eta_{i}^{n}$ is equal to 1 if sub-channel $n$ is allocated to SAP $i$; otherwise, it is equal to 0 . The instantaneous received signal-to-interference noise ratio (SINR) of UE $i$ over sub-channel $n$ can be expressed as:

$$
\gamma_{i}^{(n)}=\frac{p_{T}\left|h_{i i}^{(n)}\right|^{2}}{\sum_{j \in \mathbf{F}, j \neq i} p_{T}\left|h_{j i}^{(n)}\right|^{2}+N_{0}}
$$

where $\left|h_{j i}^{(n)}\right|^{2}$ is the channel gain from SAP $j$ to UE $i$ over sub-channel $n$ and $N_{0}$ represents the additive white Gaussian noise power. Then, the achievable rate for $F_{i}$, serving its UE $i$, is given as:

$$
R_{i}=\Delta f \log _{2}\left(1+\gamma_{i}^{(n)}\right)
$$

where $\Delta f$ is the bandwidth of sub-channel. In this paper, our objective is to find an efficient spectrum allocation scheme that maximizes the overall network throughput. We assume that each SAP can only occupy one sub-channel. Under this setting, the formulated problem is given by:

$$
\begin{aligned}
& \max _{\eta_{i}^{n}} \sum_{i \in \mathbf{F}} \eta_{i}^{n} \Delta f \log _{2}\left(1+\gamma_{i}^{(n)}\right) \\
& \text { subject to } \\
& \eta_{i}^{n} \in\{0,1\}, \forall i \in \mathbf{F}, n \in \mathbf{N}
\end{aligned},
$$

where the constraint restricts that all the elements in the sub-channel allocation matrix are $0 / 1$ variables.

Together with the objective function and pertinent constraint, we formulate an optimization problem. The objective of (3) is to find the optimal spectrum allocation $\left\{\eta_{i}^{n}\right\}, i \in\{1,2, \mathrm{~K}, K\}$ and $n \in\{1,2, \mathrm{~K}, N\}$, determining which sub-channel should be assigned to which SAP. In terms of this issue, centralized solutions are impractical due to the computational complexity in the context of large-scale networks. Distributed solutions, on the other hand, lack the superiority of centralized schemes, fading the optimization performance. Therefore, this motivates developing partially-distributed, if suboptimal, solutions.

\section{Proposed Partially-Distributed Scheme}

In response to the infeasibility of obtaining the globally optimum solution, we propose a partially-distributed spectrum allocation scheme to decompose the problem into the following three steps.

Dividing the Large-Scale Network into Disjoint Clusters. In a dense deployment of small cells with tight interference constraints, clustering is a very efficient technique which can divide the large-scale network into smaller modules, dramatically reducing the complexity of network. Since the optimal construction of the clustering is not the focus of this work, here, we adopt a simple distributed clustering method proposed in [9].

Based on clustering, all SAPs are grouped into different clusters, defined by $\mathbf{C}$. A cluster $c_{l} \in \mathbf{C}$ is the $l$ th set of small cells such that $c_{l} \in \mathbf{F}, \forall l \in\{1,2, \mathrm{~K},|\mathbf{C}|\}, \mathrm{U}_{l=1}^{\mathbf{C} \mid} c_{l}=\mathbf{F}$ and $\mathrm{I}_{l=1}^{|\mathbf{C}|} c_{l}=\phi$. Once the initial network with hyper-dense deployment of SAPs is partitioned into disjoint clusters, the spectrum 
allocation problem for overall network can be transformed to a situation in which cluster is a spectrum allocation unit.

Spectrum Allocation within Each Cluster. For a given cluster configuration, within each cluster, one SAP is elected as a $\mathrm{CH}$ that is responsible for spectrum allocation among the small cells in that cluster. We denote by $\mathrm{CH}_{l}$ the $\mathrm{CH}$ of the cluster $c_{l}$. Due to the lower coverage of SAPs, the signal transmitted by a given SAP causes interference only to the UEs located in a few neighboring cells. Thus, such local interference relationship among the small cells can be characterized by an interference graph. Here, we use a distance-determined model for presentation. The interference graph can be denoted as $G=(\mathbf{V}, \mathbf{E})$, where $\mathbf{V}$ is the set of vertices denoting SAPs and $\mathbf{E}$ is the edge set, i.e., $\mathbf{V}=\left\{v_{1}, v_{2} \mathrm{~K} v_{K}\right\}$ and $\mathbf{E}=\left\{(i, j) \mid i, j \in \mathbf{F}, d_{i j}<d_{0}\right\}$ where $d_{i j}$ is the distance between SAP $i$ and $j$, and $d_{0}$ is the threshold.

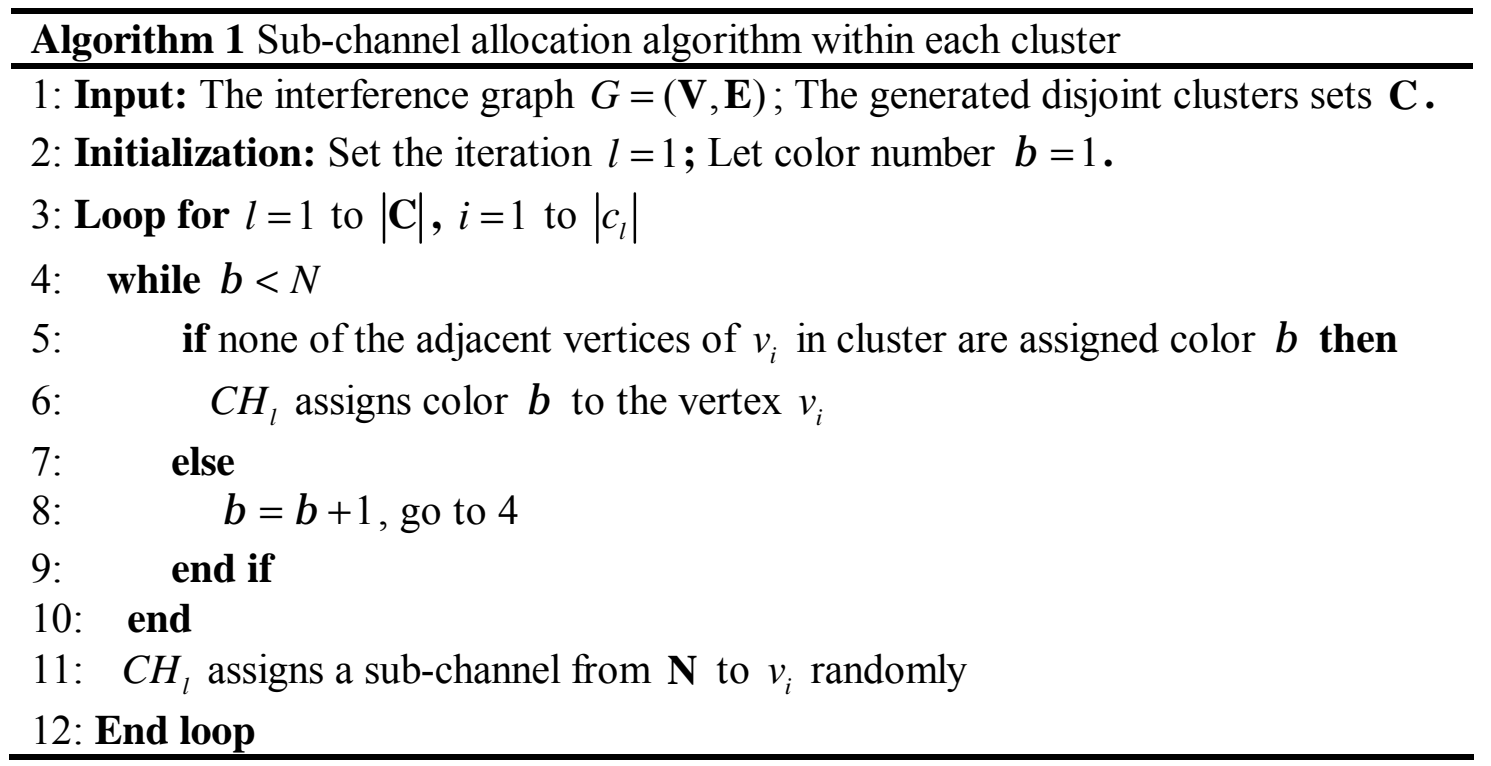

In terms of spectrum allocation within each cluster, we propose a low-complexity graph coloring based sub-channel allocation algorithm. By assuming each color as a different sub-channel, graph coloring facilitates sub-channel assignment. Let $\beta \in\{1,2 \mathrm{~K} N\}$ be the color number of vertices $v_{i}$ in $G$, and $N$ is the total number of sub-channels. The details of the sub-channel allocation algorithm are listed in Algorithm 1.

Actually, after accomplishing intra-cluster sub-channel allocation, the interference between two neighboring clusters may still exist. However, this feature was not addressed in most existing work. To tackle this issue, we present a decentralized local Q-learning scheme which is described in the next phases.

Interference Elimination among Clusters. In order to mitigate interference between two neighboring clusters, those SAPs located at the edge of clusters interfered by other neighboring SAPs from different clusters need to learn through trials and errors, and adapt the suitable channel selection strategies until resolving such interference. Instead of global distributed learning, in our scheme, a decentralized local Q-learning scheme is proposed in which only a few part of interfering SAPs in the network need to learn.

The decentralized local Q-learning algorithm for inter-cluster interference resolution is shown in Algorithm 2. In this algorithm, $N_{f}$ is the number of agents in the network which refer to the SAPs that are interfering by neighboring SAPs belonging to different clusters. Since only a small part of SAPs need to adjust channel selection strategies through learning, $N_{f}$ is generally less than $K$. $r_{t}^{i, m}=\Delta f \log _{2}\left(1+\gamma_{i}^{(m)}\right)$ is the perceived reward of SAP $F_{i}$ transmitting over sub-channel $m$ at time $t$. Each SAP performs the exploration step with probability $\varepsilon$, and $\alpha$ denotes the learning rate that is 
used to control the speed of adjustment of the Q-value. The stop criterion of the algorithm is to content that the predefined maximum iteration number is reached.

Algorithm 2 Decentralized local Q-learning algorithm for interference resolution among neighboring clusters

1: Initialization: Set the iteration index $t=0$, and the initial Q-value $\mathrm{Q}(a)=0$, for $\forall i \in\left\{1,2, \mathrm{~K} N_{f}\right\}, \forall m \in\{1,2, \mathrm{~K} M\}$.

2: Loop for $i=1$ to $N_{f}, t=0,1, \mathrm{~K}$

3: if exploration probability is less than $\varepsilon$ then

4: $\quad$ select action randomly [exploration step]

5: else

6: $\quad$ choose action $a_{t}^{i, m}=\arg \max _{a \in A} \mathrm{Q}(a)$ [exploitation step]

7: end if

8: receive immediate reward for $F_{i}$ at time $t+1: r_{t+1}^{i, m}$

9: update $\mathrm{Q}_{t+1}(a)=(1-\alpha) \mathrm{Q}_{t}(a)+\alpha\left(r_{t}(a)+\lambda \max _{a^{\prime} \in A} \mathrm{Q}_{t}\left(a^{\prime}\right)\right)$

10: End Loop

\section{Simulation Results}

In this section, simulation results are presented to show the effectiveness of the proposed partially-distributed spectrum allocation scheme. Here, we consider a hyper-dense small cell network with large-scale deployment of femtocells in which the femtocell access points (FAPs) are randomly distributed in a 2-D $100 \mathrm{~m} \times 100 \mathrm{~m}$ area and each FAP can cover a circular cell region of radius 10 meters. The bandwidth of each sub-channel is $180 \mathrm{kHz}$. The transmission power of the FAPs is set to $20 \mathrm{dBm}$. We set the discount factor $\lambda=0.3$ and the learning rate $\alpha=0.3$ for the decentralized local Q-learning stage. The channel gains include path-loss and shadowing. For path-loss between a femtocell and its UE, $P L=38.46+20 \log d$ and for path-loss between a femtocell and a general UE $P L=38.46+20 \log d+q L, d$ is the distance between a FAP and the UE and $q L$ accounts for loss due to walls [10]. Standard deviation of shadowing between a FAP and its attaching UE is $4 \mathrm{~dB}$, while for other UEs is $8 \mathrm{~dB}$.

Convergence Behavior. In this subsection, we present the convergence behavior of the proposed partially-distributed scheme. We consider a relatively large-scale small cell network with hyper-dense deployment of femtocells consisting of 50 FAPs. The inner wall loss is set to $10 \mathrm{~dB}$ and 5 sub-channels are available in the network. In this context, the convergence behavior for the proposed scheme is shown in Fig. 1. This result is obtained by taking the expected value of 100 independent trials. It is note that the proposed scheme converges in about 350 iterations. This result validates the convergence of the proposed scheme in the context of large-scale networks.

Performance Comparison. In this subsection, the performance comparison results are presented. We compare our proposed scheme with three existing baseline approaches for spectrum allocation in small cell networks: distributed global learning algorithm [11], graph-based clustering resource allocation scheme [12] and random allocation scheme.

Fig. 2 shows the variation in system throughput with the number of FAPs. We change the FAP number from 50 to 80 , corresponding to the increase of femtocell density. We observe that our proposed partially-distributed scheme offers a higher system throughput in comparison to the graph-based clustering resource allocation and random allocation schemes. Although distributed global learning algorithm has a performance that is close to our proposed scheme and better than the other two methods, it needs a large number of trials and errors for each FAP to select the suitable strategy. 


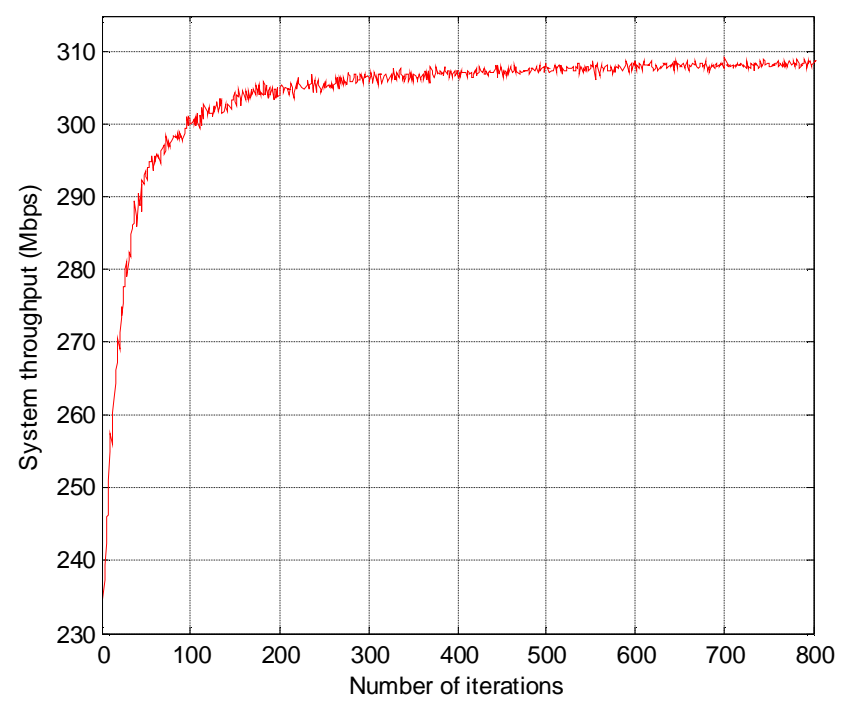

Fig 1. Convergence behavior of the proposed partially-distributed scheme.

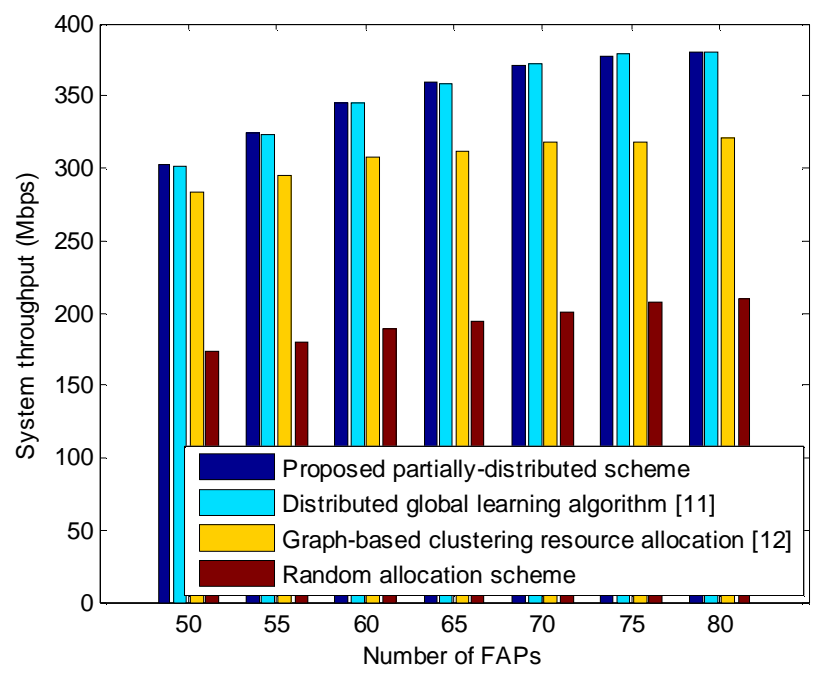

Fig. 2. Total throughput of the system versus the number of FAPs.

In order to study the convergence speeds of the distributed global learning algorithm and our proposed scheme for different network scenarios, we compare the convergence time towards these two schemes, from a statistical perspective. Specifically, the convergence speeds of the two schemes are shown in Fig. 3. The convergence time for a given network scale is obtained by taking the expected value of 500 independent trials. It is noted from the figure that the convergence speed of our method is faster than that of the distributed global learning algorithm as expected.

Summarizing the above simulation results, we can see that our proposed partially-distributed scheme can obtain better performance in the aspects of throughput than the existing spectrum allocation solutions such as graph-based clustering resource allocation and random allocation schemes. Although the proposed scheme is close to the distributed global learning algorithm in term of throughput performance, the convergence speed of our method is much faster than the distributed global learning algorithm, especially in large-scale networks, that will be more obvious. Therefore, the proposed partially-distributed scheme has more advantages than some other existing methods for spectrum allocation in hyper-dense networks. 


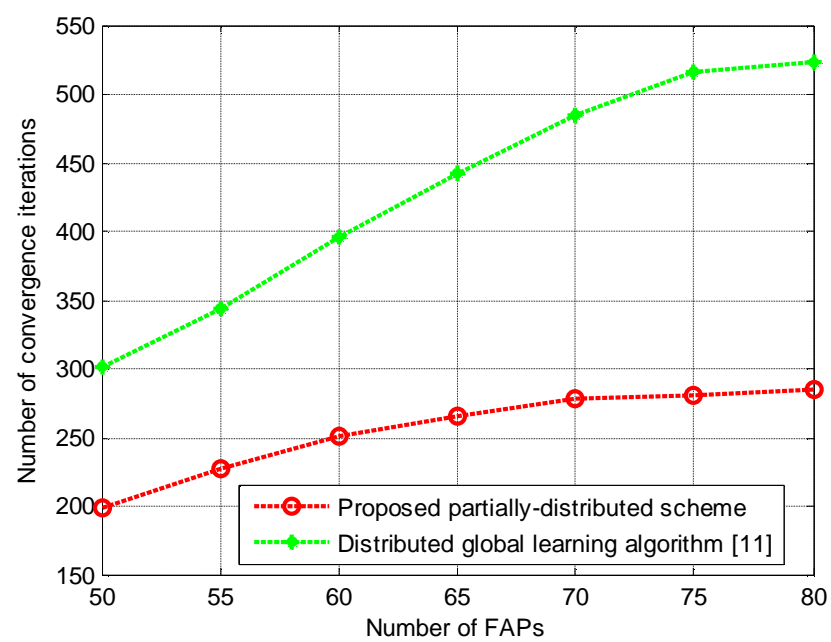

Fig. 3. Convergence speeds of the proposed partially-distributed scheme and distributed global learning algorithm for different network scales.

\section{Conclusions}

In this paper, we have investigated spectrum allocation problem for hyper-dense small cell networks. Since obtaining the globally optimum spectrum allocation is very complex and mathematically intractable, we attempt to give a practical partially-distributed spectrum allocation scheme while getting a desirable solution. The main advantage of the proposed scheme is decomposing a complexity optimization problem into three phases with controllable time and computation complexities. The numerical results reveal that the proposed partially-distributed scheme can get better tradeoff in computation complexity and achieved performance compared to other existing baseline approaches. Our proposed scheme is a heuristic method so the results may not be the optimal. In the future, we can consider the optimization approach to improve the performance further.

\section{References}

[1] J. Xu, J. Wang, Y. Zhu, Y. Yang, X. Zheng, S. Wang, et al., Cooperative distributed optimization for the hyper-dense small cell deployment, IEEE Commun. Mag. 52 (2014) 61-67.

[2] H. Hsieh, S. Wei and C. Chien, Optimizing small cell deployment in arbitrary wireless networks with minimum service rate constraint, IEEE Trans. Mobile Comput. 13 (2014) 1801-1815.

[3] J. Kim and D.H. Cho, A joint power and subchannel allocation scheme maximizing system capacity in indoor dense mobile communication systems, IEEE Trans. Veh. Technol. 59 (2010) 4340-4353.

[4] K. Zheng, J. Wang, C. Lin, X. Shen and Y. Wang, Graph-based interference coordination scheme in orthogonal frequency-division multiplexing access femtocell networks, IET Commun. 5 (2011) 2533-2541.

[5] L. Li, C. Xu and M. Tao, Resource allocation in open access OFDMA femtocell networks, IEEE Wirel. Commun. Lett. 1 (2012) 625-628.

[6] F. Pantisano, M. Bennis, W. Saad, M. Debbah and M. Latva-aho, Interference alignment for cooperative femtocell networks: a game theoretic approach, IEEE Trans. Mobile Comput. 12 (2013) 2233-2246.

[7] S. Lin, and H. Tian, Clustering based interference management for QoS guarantees in OFDMA femtocell, in Proc. of IEEE Wireless Communications and Networking Conference (WCNC), Shanghai, 2013, pp. 649-654. 
[8] S. J. Kim, and I. Cho, Graph-based dynamic channel assignment scheme for femtocell networks, IEEE Commun. Lett. 17 (2014) 1718-1721.

[9] A. Hatoum, N. Aitsaadi, R. Langar, R. Boutaba and G. Pujolle, FCRA: femtocell cluster-based resource allocation scheme for OFDMA networks, in Proc. of IEEE International Conference on Communications (ICC), Kyoto, 2011, pp. 1-6.

[10]A. Abdelnasser, E. Hossain and D. I. Kim, Clustering and resource allocation for dense femtocells in a two-tier cellular OFDMA network, IEEE Trans. Wireless Commun. 13 (2014) 1628-1641.

[11]M. Bennis, and D. Niyato, A Q-learning based approach to interference avoidance in self-organized femtocell networks, in Proc. of IEEE Globecom Workshops (GC Wkshps), Miami, 2010, pp. 706-710.

[12]Q. Zhang, X. Zhu, L. Wu and K. Sandrasegaran, A coloring-based resource allocation for OFDMA femtocell networks, in Proc. of IEEE Wireless Communications and Networking Conference (WCNC), Shanghai, 2013, pp. 673-678. 